\title{
NueVas citas de macrolíquenes para Argentina y ampliaciones DE DISTRIBUCIÓN EN EL CENTRO DEL PAÍS
}

\author{
JUAN MANUEL RODRIGUEZ ${ }^{1 *}$, JUAN MARTIN HERNANDEZ², EDITH FILIPPINI ${ }^{1}$, MARTHA \\ CAÑAS ${ }^{2}$ y CECILIA ESTRABOU ${ }^{1}$
}

\begin{abstract}
Summary: New records of macrolichens and increasing distributional range in central Argentina. Four species of lichenized Ascomycetes are mentioned for the first time from Argentina: Endocarpon pallidulum, Placidium arboreum, Pyxine astridiana and Usnea michauxii. A brief description of each one is presented considering morphological, anatomical and chemical characteristics. The distribution of 68 lichen species in Argentina is also extended.
\end{abstract}

Key words: Diversity, taxonomy, lichenized Ascomycetes, South America.

Resumen: Se mencionan por primera vez para el país cuatro especies de Ascomycetes liquenizados: Endocarpon pallidulum, Placidium arboreum, Pyxine astridiana y Usnea michauxii. Se presenta una breve descripción de cada una considerando características morfológicas, anatómicas y químicas. A su vez se amplía la distribución de 68 especies de líquenes en el centro de Argentina.

Palabras clave: Diversidad, taxonomía, Ascomycetes liquenizados, Sudamérica.

\section{INTRODUCCIÓN}

La diversidad de líquenes en Argentina ha sido motivo de numerosas revisiones y actualizaciones. Desde que se publicó el último catálogo de líquenes para el país (Calvelo \& Liberatore, 2002) algunos grupos o géneros de macrolíquenes han sido profundamente trabajados (Filippini et al., 2015; Rodríguez et al., 2011; Ferraro \& Michlig, 2013; Passo \& Calvelo, 2006; Passo et al., 2008) y se han publicado otras novedades taxonómicas (Estrabou et al., 2006; Michlig \& Ferraro, 2012; Adler \& Calvelo, 2010).

El centro de Argentina se corresponde con un gradiente climático de dirección NE - SO que marca diferencias en precipitaciones y temperatura que, en

1 IIBYT (CONICET - Universidad Nacional de Córdoba) y CERNAR (FCEFyN - UNC). Av. Velez Sarsfield 1611. Córdoba.

2 CITCA (CONICET - Universidad Nacional de Catamarca) y Facultad de Tecnología y Ciencias Aplicadas, Universidad Nacional de Catamarca. * Autor para correspondencia: juanmacor@yahoo.com.ar consecuencia, genera una diversidad de formaciones vegetales que se traduce en provincias fitogeográficas (Cabrera, 1971). Los territorios de llanura del centro del país son parte de las unidades del Chaco, Espinal y Monte. A su vez, las Sierras Pampeanas constituyen un importante gradiente alitudinal y se transforman en un reservorio de biodiversidad a causa de las notables diferencias climáticas que ocurren a medida que la altitud aumenta. La unidad fitogeográfica que ocupa las montañas del centro de país es el Chaco Serrano. Esta vasta y diversa región ha sido pobremente explorada para la colección y estudio de los líquenes. Por este motivo el inicio de trabajos sobre la diversidad y taxonomía de estos organismos en el área de estudio da como primer resultado la ampliación del rango de distribución de numerosas especies. Esta información de base es relevante para futuros estudios en ecología y para la conservación de los líquenes y de los ambientes de los cuales dependen.

En este trabajo se describen cuatro especies como nuevas para el país y se amplía el rango de distribución de 68 especies de macrolíquenes previamente conocidas para Argentina. 


\section{Materiales y Métodos}

Se estudiaron especímenes coleccionados recientemente por los autores en las provincias de Catamarca, Córdoba, La Rioja, San Luis, Santiago del Estero y Tucumán, de los cuales un ejemplar de cada especie por provincia se encuentra depositado en el herbario CORD. La metodología utilizada para la identificación del material incluye métodos de rutina para el trabajo en taxonomía de líquenes: análisis morfológico (macro y microscópico), anatómico y químico. Para la observación de las esporas y conidios se realizaron cortes a mano alzada de los apotecios y picnidios, que fueron posteriormente montados en hidróxido de potasio al 5\% para ser observados en microscopio óptico.

La identificación de las sustancias liquénicas presentes fue realizada a través de reacciones puntuales de color con hidróxido de potasio al $10 \%(\mathrm{~K})$, hipoclorito de sodio en concentración comercial (C), $\mathrm{K}$ luego $\mathrm{C}$ (KC), fluorescencia en cámara de luz ultravioleta y Cromatografía en Capa Delgada (CCD) de acuerdo a la metodología propuesta por Orange et al. (2010).

Los resultados se presentan en dos apartados: Nuevas citas para Argentina con la descripción completa de cuatro especies y Ampliaciones de distribución en Argentina. Para éstas últimas se cita un artículo que contiene una descripción completa. Los datos de distribución en Argentina pueden consultarse en Calvelo \& Liberatore (2002) y en trabajos posteriores citados en la bibliografía de cada especie. Se cita un ejemplar de cada especie por provincia, tanto para las ampliaciones como para las nuevas citas.

\section{Resultados}

Nuevas citas para Argentina

Endocarpon pallidulum (Nyl.) Nyl., Nouv. Arch. Mus. Hist. Nat. 4: 106. 1892. Figura 1A.

Talo escuamuloso, corticícola, beige a marrón claro, escuámulas de 1-3 mm de ancho, levemente convexas y a veces superpuestas. Superficie superior lisa, algo brillante. Soralios ausentes. Médula blanca, capa algal gruesa. Superficie inferior marrón claro, con rizohifas hialinas. Peritecios con ostíolo claro (poco visible) e himenio con algas. Ascos globosos, ascósporas muriformes, 2 por

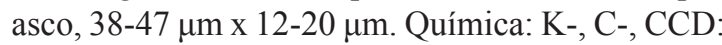
negativa. Esta especie tiene una distribución amplia en todo el mundo, en Sudamérica se registró en Perú y Brasil (GBIF, 2015).

Material estudiado: ARGENTINA. Prov. Córdoba: Dpto. Juárez Celman, Alejandro Roca, S $33^{\circ} 18^{\prime} 31,80^{\prime \prime}$; O $63^{\circ} 45^{\prime}$ 6,12'", 215 m, sobre Celtis erhenbergiana, III-2014, Filippini 4078 (CORD).

Placidium arboreum (Schwein. ex E. Michener) Lendemer, Mycotaxon 90 (2): 320. 2004. Figura 1B

Talo escuamuloso, corticícola, marrón claro a marrón oscuro en los bordes, escuámulas de 5-10 $\mathrm{mm}$ de ancho, lobuladas, solapadas entre sí y cóncavas. Superficie superior lisa, sin brillo. Soralios ausentes. Médula blanca, capa algal gruesa. Superficie inferior clara a amarronada, con rizohifas hialinas a amarronadas, agrupadas en cepillos. Peritecios con ostíolos oscuros y sin algas en el himenio. Ascos cilíndricos, ascósporas elipsoidales uniseriadas, simples, hialinas, 9-13 $\mu \mathrm{m}$ x 4-16 $\mu \mathrm{m}$. Química: K-, C-, CCD: negativa.

Placidium arboreum sólo ha sido registrada en Norte y Centro América (Lendemer \& Yahr, 2004).

Material estudiado: ARGENTINA, Prov. Córdoba: Dpto. General San Martín, Estancia

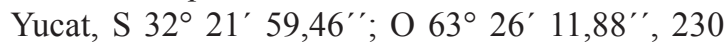
m, sobre Prosopis sp., V-2014, Filippini 4079 (CORD).

Pyxine astridiana Kalb, Bibl. Lichenol.24: 33.1987. Figura 1C

Talo folioso, corticícola, hasta $8 \mathrm{~cm}$ de diám., grisáceo a gris verdoso en el centro, fuertemente adherido al sustrato. Lóbulos de 1-1,5 mm de ancho, con ápices redondeados y a veces cóncavos, ligeramente superpuestos. Superficie superior maculada, con pruina fina sobre los lóbulos marginales. Soralios ausentes. Médula blanca. Superficie inferior negra a grisácea en los márgenes, con ricines simples, cortos, concoloros. Apotecios de tipo physciaeformis, hasta $1,5 \mathrm{~mm}$ de diám., sésiles, con disco negro y margen crenado 


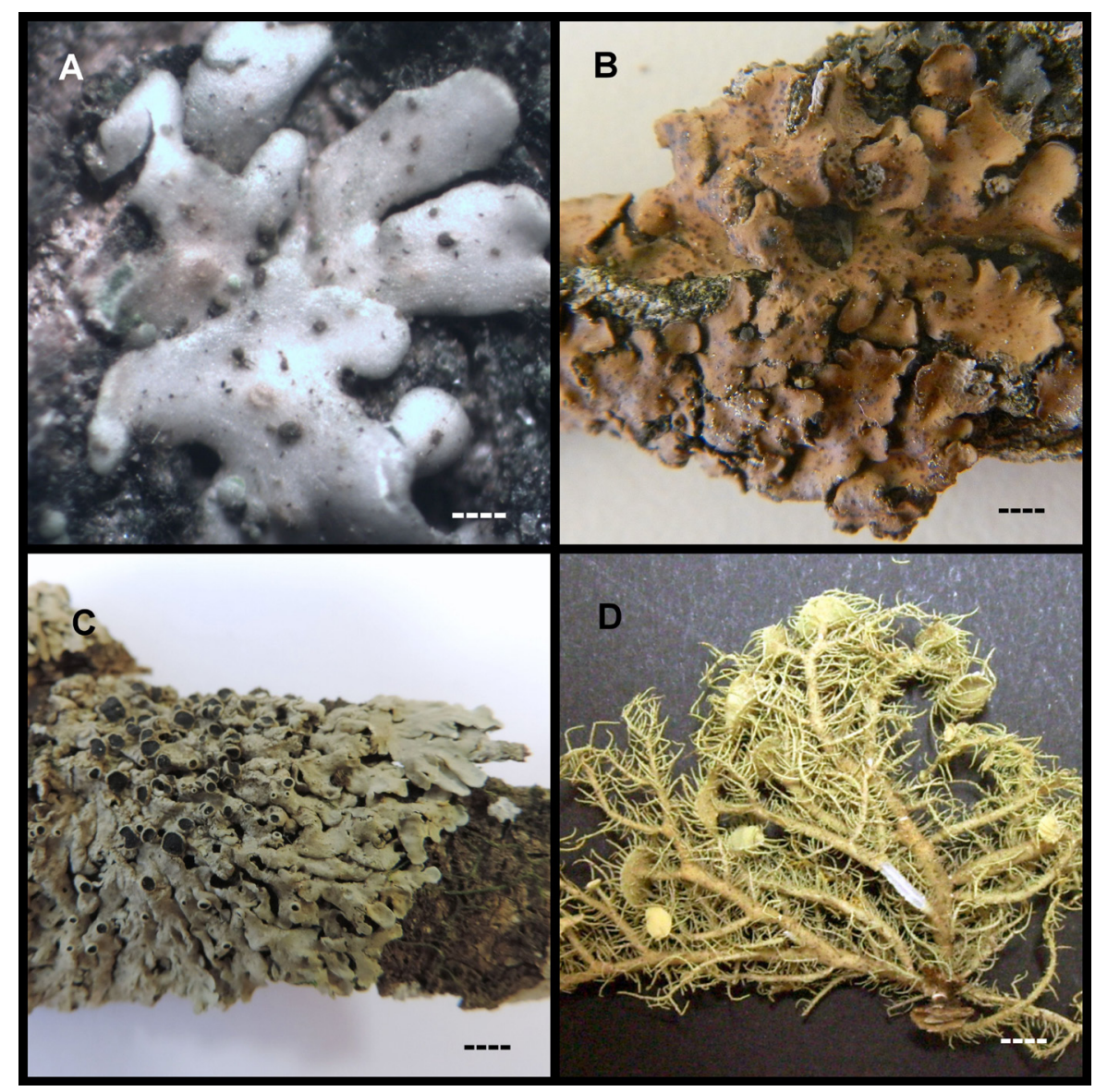

Fig. 1. Especies citadas por primera vez en Argentina. A:Endocarpon pallidulum. B:Placidium arboreum. C: Pyxine astridiana. D:Usnea michauxii. Escalas: $A=0,37 \mathrm{~mm} ; B=1 \mathrm{~mm} ; C=1,2 \mathrm{~mm} ; \mathrm{D}=2 \mathrm{~mm}$.

en apotecios maduros. Ascósporas 1-septadas, de 17-18 $\mu \mathrm{m}$ x 6-7 $\mu \mathrm{m}$. Picnidios no vistos.

Química: corteza superior K-, UV+ amarillo; médula $\mathrm{K}+$ amarillo suave, $\mathrm{C}-, \mathrm{CCD}$ : sustancia desconocida, amarillo claro, Rf: 3

Observaciones: Jungbluth (2010) describe a Pyxine astridiana con médula $\mathrm{K}$ - sin embargo las muestras observadas en este trabajo coinciden morfológica y anatómicamente con esta especie con excepción de la reacción de $\mathrm{K}$ en la médula que es amarillo pálido.

Material estudiado: ARGENTINA. Prov. Catamarca:Dpto. Andalgalá, S $27^{\circ} 29^{\prime} 4,13^{\prime \prime}$; O 66 31' 28,60"' 1489 m, sobre Prosopis sp., VII2014, Filippini 3100 (CORD).
Usnea michauxii I. Tavares, Mycotaxon 30: 54. 1987. Figura 1D

Talo fruticuloso erecto, hasta $4 \mathrm{~cm}$ de largo, color verde grisáceo con puntos rojos a lo largo del todo el talo. Ramas fusiformes a irregulares marcadamente segmentadas; segmentos inflados y en sección transversal circulares; ramas secundarias constrictas en el punto de ramificación; fibrilas numerosas, espinulosas, cortas, hasta $2 \mathrm{~mm}$ de longitud, irregularmente distribuidas. Soralios e isidiomorfos ausentes. Corteza con puntos rojos en todo el talo, brillante y quebradiza, muy delgada. Médula muy laxa, aracnoidea y gruesa, eje recto y delgado. Apotecios terminales, subterminales o laterales, con fibrilas marginales, disco pruinoso. 
Ascósporas simples y hialinas, $8,7 \mu \mathrm{m}$ x 5,7 $\mu \mathrm{m}$ en promedio. Picnidios presentes en ramas terminales y fibrilas, conidios sublageniformes $6,75 \mu \mathrm{m}$ de longitud promedio. Química: K-, C-, CCD: ácido úsnico y ácidos grasos no identificados. Esta especie ha sido descripta para Estados Unidos (Tavares, 1987), Bolivia y Perú (Truong et al., 2011).

Material estudiado: ARGENTINA. Prov. Córdoba: Dpto. Punilla, Los Gigantes, S $31^{\circ} 25^{\prime}$ 39,50"'; O 64 45'48,60", sobre poste, Rodríguez 386 (CORD). Prov. Tucumán: Dpto. Yerba Buena, Reserva San Javier, S $26^{\circ} 46^{\prime} 0,50^{\prime \prime}$; O $65^{\circ} 19^{\prime}$ 71,00", 723 m, Rodríguez 158 (CORD).

\section{Ampliaciones de distribución en Argentina}

Candelaria concolor (Dicks.) Arnold, Cohn's Krypt.-Flora von Schlesien: 364. 1879.

Bibliografía: Wetsberg \& Nash (2002).

Amplía su distribución a la provincia de Catamarca. Material estudiado: ARGENTINA. Prov. Catamarca: Dpto. Andalgalá, RP1 al lado de arroyo, S $27^{\circ} 39^{\prime}$ 2,40"'; O 65 56' 47,40", $1940 \mathrm{~m}$, sobre roca, 11-VII-2011, Rodríguez 2710 (CORD).

Crespoa carneopruinata (Zahlbr.) Lendemer \& Hodkinson, N. Am. Fungi, 7 (2): 3. 2012.

Bibliografía: Michlig (2014).

Amplía su distribución a la provincia de Catamarca. Material estudiado: ARGENTINA. Prov. Catamarca: Dpto. Santa Rosa, a $10 \mathrm{~km}$ de Bañado de Ovanta (dirección este), S $28^{\circ} 07^{\prime} 46,30^{\prime \prime}$; O 65 $15^{\circ}$ $47,10^{\prime \prime}, 523 \mathrm{~m}$, sobre ramas, 12-VII-2011, Rodríguez 2601 (CORD).

Observaciones: citada previamente en el país como Canoparmelia carneopruinata.

Crespoa crozalsiana (B. de Lesd.) Lendemer \& Hodkinson, N. Am. Fungi 7 (2): 3. 2012.

Bibliografía: Michlig (2014).

Amplía su distribución a la provincia de Catamarca.

Material estudiado: ARGENTINA. Prov. Catamarca: Dpto. Ambato, La Calera, S 28 $22^{\prime}$ $12,90^{\prime \prime}$; O $65^{\circ} 52^{\prime} 10,40^{\prime \prime}, 1154 \mathrm{~m}$, sobre ramas, 11-VII-2011, Rodríguez 2649 (CORD).

Observaciones: citada previamente en el país como Canoparmelia crozalsiana.

Culbersonia nubila (Moberg) Essl., Lich. Fl.
Gr. Sonoran Desert Region 1: 164. 2002.

Bibliografía: Obermayer et al. (2009).

Amplía su distribución a la provincia de Catamarca.

Material estudiado: ARGENTINA. Prov. Catamarca: Dpto. Andalgalá, Qda. de Vis-Vis, DS6, S $27^{\circ} 27^{\prime} 22,47^{\prime \prime}$; O $66^{\circ} 31^{\prime} 18,90^{\prime \prime}$, 1700 m, sobre Larrea divaricata, 10-VIII-2015, Rodríguez 3120 (CORD). Prov. Córdoba: Dpto. Punilla, Los Gigantes, Cuesta del perro, S $31^{\circ} 24^{\prime} 11,2^{\prime \prime}$; O $64^{\circ} 48^{\prime}$ 01,5"', 1960 m, sobre suelo, III-2015, Rodriguez 4082 (CORD).

Observaciones: especie transferida al género Culbersonia a partir de Pyxine nubila en base a su pigmentación azulada en la corteza que es $\mathrm{K}+$ púrpuray al tamaño y forma de los conidios (5 - 6,5 $\mu \mathrm{m}$, cilíndricos a levemente fusiformes).

Flavoparmelia caperata (L.) Hale, Mycotaxon 25 (2): 604. 1986.

Bibliografía: Estrabou (1999).

Amplía su distribución a la provincia de Catamarca.

Material estudiado: ARGENTINA. Prov. Catamarca: Dpto. Ambato, Sierras de Ambato, $\mathrm{S} 28^{\circ} 00^{\prime} 41,20^{\prime \prime}$; O $65^{\circ} 56^{\prime} 38,10^{\prime \prime}, 1684 \mathrm{~m}$, sobre tierra y musgos, 14-X-2007, Rodríguez 2905 (CORD).

Flavoparmelia exornata (Zahlbr.) Hale, Mycotaxon 25 (2): 604. 1986.

Bibliografía: Estrabou (1999).

Amplía su distribución a la provincia de Catamarca.

Material estudiado: ARGENTINA. Prov. Catamarca: Dpto. Andalgalá, Qda. de Vis-Vis, DS6, S $27^{\circ} 27^{\prime} 22,47^{\prime \prime}$; O $66^{\circ} 31^{\prime} 18,90^{\prime \prime}, 1700$ m, sobre Larrea divaricata, 10-VIII-2015, Rodríguez 4081 (CORD).

Flavoparmelia soredians (Nyl.) Hale, Mycotaxon 25 (2): 605. 1986.

Bibliografía: Estrabou (1999).

Amplía su distribución a la provincia de Catamarca.

Material estudiado: ARGENTINA. Prov. Catamarca: Dpto. Andalgalá, Qda. de Vis-Vis, DS6, S $27^{\circ} 27^{\prime} 22,47^{\prime \prime}$; O 66 31' 18,90"', 1700 m, sobre Larrea divaricata, 10-VIII-2015, Rodríguez 4080 (CORD). 
Flavopunctelia flaventior (Stirt.) Hale, Mycotaxon 20 (2): 682. 1984.

Bibliografía: Estrabou (1999).

Amplía su distribución a la provincia de Tucumán.

Material estudiado: ARGENTINA. Prov. Tucumán:Dpto. Tafí, RP 307 a $20 \mathrm{Km}$ de empalme

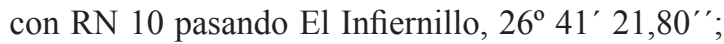
O $65^{\circ} 48^{\prime} 32,70^{\prime \prime}, 2875 \mathrm{~m}$, sobre arbusto, 10-VII2011, Rodríguez 2659 (CORD).

Heterodermia diademata (Taylor) D.D. Awasthi, Geophytology 3 (1):113. 1973.

Bibliografía: Rodríguez et al. (2012).

Amplía su distribución a la provincia de Tucumán.

Material estudiado: ARGENTINA. Prov. Tucumán:Dpto. Yerba Buena, RP 340 pasando San Javier al lado del camino, S 26 46' 15,60"' $65^{\circ} 21^{\prime} 10,60^{\prime \prime}, 1180 \mathrm{~m}$, sobre rama, 11-VII-2011, Rodríguez 2548 (CORD).

Heterodermia fertilis Moberg, Nord. J. Bot. 29: 136. 2011.

Bibliografía: Rodríguez et al. (2012).

Amplía su distribución a la provincia de Tucumán. Material estudiado: ARGENTINA. Prov. Tucumán:Dpto. Chicligasta, límite TucumánCatamarca (cuesta del Clavillo), S $27^{\circ} 20^{\prime} 2,00^{\prime \prime}$; O 65 56' 52,80", 1790 m, sobre Alnus acuminata, 11-VII-2011, Rodríguez 2575 (CORD).

Heterodermia flabellata (Fée) D.D. Awasthi, Geophytology 3: 113. 1973.

Bibliografía: Rodríguez et al. (2012).

Amplía su distribución a la provincia de Tucumán. Material estudiado: ARGENTINA. Prov. Tucumán: Dpto. Yerba Buena, RP 340 San Javier, S 26 46' 34,00'"; O 65 22' 34,40"', 1077 m, sobre Alnus acuminata, 11-VII-2011, Rodríguez 2554 (CORD).

Heterodermia leucomela (L.) Poelt, Nova Hedwig. Beih. 9: 31. 1965.

Bibliografía: Rodríguez et al. (2012).

Amplía su distribución a la provincia de Catamarca.

Material estudiado: ARGENTINA. Prov. Catamarca: Dpto. Paclin, a $10 \mathrm{~km}$ de La Merced, S $28^{\circ} 06^{\prime} 1,60^{\prime \prime}$; O $65^{\circ} 36^{\prime} 45,90^{\prime \prime}, 983 \mathrm{~m}, 12-\mathrm{VII}-$ 2011, Rodríguez 2604 (CORD).
Hypotrachyna bonariensis (Adler \& Elix) Divakar, A. Crespo, Sipman, Elix \& Lumbsch, Phytotaxa 132 (1): 33. 2013.

Bibliografía: Adler \& Elix (1987).

Amplía su distribución a la provincia de Córdoba.

Material estudiado: ARGENTINA. Prov. Córdoba: Dpto. Calamuchita, Camino de los Linderos,S $32^{\circ} 05^{\prime}$ 9,40"'; O 64 50'30,60", 1367 $\mathrm{m}$, sobre roca, 30-VIII-2013, Rodríguez 3052 (CORD).

Observaciones: especie descubierta en Argentina como Parmelinopsis bonariensis.

Hypotrachyna brevirhiza (Kurok.) Hale, Smithsonian Contr. Bot. 25: 26. 1975.

Bibliografía: Calvelo (1994).

Amplía su distribución as las provincias de Catamarca, Córdoba y Tucumán.

Material estudiado: ARGENTINA. Prov. Catamarca: Dpto. Andalgalá, RP 1 al límite con Tucumán, S $27^{\circ} 20^{\prime}$ 38,30"'; O $65^{\circ} 57^{\prime}$ 59,70', 1896 m, sobre Alnus acuminata, 11-VII-2011, Rodríguez 3069 (CORD). Prov. Córdoba: Dpto. Punilla, Los Gigantes,S 31 $24^{\prime}$ 8,30' ' O 64 $44^{\circ}$ 43,60", $2261 \mathrm{~m}$, sobre arbustos secos, 06-III-2015, Rodríguez 3076 (CORD). Prov. Tucumán:Dpto.

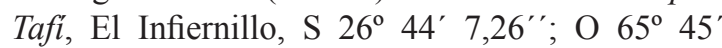
47,00", 3033 m, 10-VII-2011, Rodríguez 2612 (CORD).

Hypotrachyna cirrhata (Fries) Divakar, A. Crespo, Sipman, Elix \& Lumbsch, Phytotaxa 132 (1): 31. 2013.

Bibliografía: Calvelo \& Estrabou (1997).

Amplía su distribución a la provincia de Catamarca.

Material estudiado: ARGENTINA. Prov. Catamarca: Dpto. Ambato, Las Chacritas (a $10 \mathrm{~km}$ de Singuil), S $27^{\circ} 42^{\prime}$ 39,40"'; O $65^{\circ} 54^{\prime} 19,60^{\prime \prime}$, 1838 m, sobre aliso, 11-VII-2011, Rodríguez 2624 (CORD).

Observaciones: especie citada en Argentina como Cetrariatrum cirrhatum.

Hypotrachyna leiophylla (Kurok.) Hale, Phytologia 28 (4): 341. 1974.

Bibliografía: Adler \& Elix (1992).

Amplía su distribución a la provincia de Córdoba.

Material estudiado: ARGENTINA. Prov. Córdoba: Dpto. Calamuchita, Camino de los 
Linderos, S 32 $04^{\prime} 23,80^{\prime \prime}$; O 64 51' 58,90", 1620 m,sobre roca, 30-VIII-2013, Rodríguez 3048 (CORD).

Hypotrachyna livida (Taylor) Hale, Smithsonian Contr. Bot. 25: 45. 1975.

Bibliografía: Adler \& Elix (1992).

Amplía su distribución a la provincia de Catamarca.

Material estudiado: ARGENTINA. Prov. Catamarca: Dpto. Andalgalá, RP 1 al límite con Tucumán, S $27^{\circ} 20^{\prime}$ 38,30'"; O 65 57' 59,70", 1896 m, sobre Alnus acuminata, 11-VII-2011, Rodríguez 3098 (CORD).

Hypotrachyna subpustulifera Elix, Mycotaxon 47: 108. 1993.

Bibliografía: Michlig (2013).

Amplía su distribución a la provincia de Córdoba.

Material estudiado: ARGENTINA. Prov. Córdoba: Dpto. Calamuchita, Camino de los Linderos, S 32 $04^{\prime}$ 23,80"; O 64 51' 58,90", 1620 m, sobre roca, 30-VIII-2013, Rodríguez 3061 (CORD).

Myelochroa lindmanii (Lynge) Elix \& Hale, Mycotaxon 29: 241. 1987.

Bibliografía: Adler (2013).

Amplía su distribución a la provincia de Tucumán.

Material estudiado: ARGENTINA. Prov. Tucumán: Dpto. Yerba Buena, Camino a San Javier

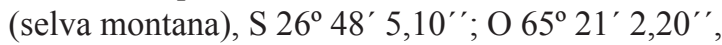
1007 m, sobre ramas, 11-VII-2011, Rodríguez 2557 (CORD).

Parmotrema austrosinense (Zahlbr.) Hale, Phytologia 28 (4): 335. 1974.

Bibliografía: Adler (2013).

Amplía su distribución a la provincia de Catamarca.

Material estudiado: ARGENTINA. Prov. Catamarca: Dpto. Santa Rosa, ruta 64 (Bosque Chaqueño de llanura), S $28^{\circ} 06^{\prime} 6,60^{\prime \prime}$; O $65^{\circ} 22^{\prime}$ 40,90", 510 m, sobre Acacia sp., 11-VII-2011, Rodríguez 2591 (CORD).

Parmotrema conferendum Hale, Mycotaxon 5 (2): 433.1977.

Bibliografía: Adler (2013).
Amplía su distribución as las provincias de Catamarca y Tucumán.

Material estudiado: ARGENTINA. Prov. Catamarca: Dpto. Ambato, El Rodeo, S 28 13'; O 65 53' 13,30", Rodríguez \& Estrabou 2685 (CORD). Prov. Tucumán: Dpto. Yerba Buena,

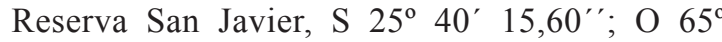
$21^{\prime} 10,60^{\prime \prime}, 1180 \mathrm{~m}$, sobre poste, 09-VII-2011, Rodríguez 2591 (CORD).

Parmotrema consors (Nyl.) Krog \& Swinscow, Lichenologist 15 (2): 129. 1983.

Bibliografía: Adler (2013).

Amplía su distribución as las provincias de Catamarca y Tucumán.

Material estudiado: ARGENTINA. Prov. Catamarca: Dpto. Ambato, Colpes (Río Los Puestos),

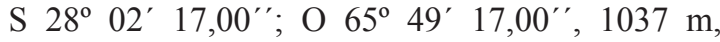
sobre corteza, Rodríguez 2695 (CORD). Prov. Tucumán:Dpto. Tafi Viejo, RP $3417 \mathrm{~km}$ antes de empalme con RN 9, S $26^{\circ} 37^{\prime} 13,80^{\prime \prime}$; O 65 $21^{\circ}$ 31,00", 879 m, sobre Prosopis sp., 10-VII-2011, Rodríguez 2670 (CORD).

Observaciones: especie citada en Argentina como Canomaculina consors.

Parmotrema pilosum (Stizenb.) Krog \& Swinscow, Lichenologist 15 (2): 130. 1983.

Amplía su distribución as las provincias de Catamarca y Tucumán.

Bibliografía: Estrabou (1999).

Material estudiado: ARGENTINA. Prov. Catamarca: Dpto. Valle Viejo, Portezuelo, S 28 $27^{\circ}$ 28,20"'; O 65 37' 37,20", 647 m, 12-VII-2011, Rodríguez 2698 (CORD).Prov. Tucumán: Dpto. Tafi, RP 307 pasando Reserva Los Sosa (selva), S 27 05' 29,20"'; O 65 39' 34,80", $723 \mathrm{~m}$, sobre ramas, 10VII-2011, Rodríguez 2662 (CORD).

Observaciones: especie citada en Argentina como Canomaculina pilosa.

Parmotrema praesorediosum (Nyl.) Hale, Phytologia 28 (4): 338. 1974.

Bibliografía: Adler (2013).

Amplía su distribución a la provincia de Catamarca. Material estudiado: ARGENTINA. Prov. Catamarca: Dpto. Santa Rosa, a $10 \mathrm{~km}$ de bañado de Ovanta (dirección este), S $28^{\circ} 07^{\prime} 46,30^{\prime \prime}$; O 65 $15^{\prime}$ 47,10", $523 \mathrm{~m}$, sobre ramas, 12-VII-2011, Rodríguez 2540 (CORD). 
Parmotrema reticulatum (Taylor) M. Choisy, Bull. Mens. Soc. Linn. Lyon 21: 175. 1952.

Bibliografía: Adler (2013).

Amplía su distribución a la provincia de Catamarca.

Material estudiado: ARGENTINA. Prov. Catamarca: Dpto. Ambato, Colpes (Río Los Puestos), S $28^{\circ} 02^{\prime} 17,00^{\prime \prime}$; O $65^{\circ} 49^{\prime} 17,00^{\prime \prime}, 1037$ m, sobre corteza, 12-VII-2011, Rodríguez 2561 (CORD).

Observaciones: especie citada en Argentina como Rimelia reticulata.

Parmotrema tandilense (Adler \& Elix) O. Blanco, A. Crespo, Divakar, Elix \& Lumbsch, Mycologia 97(1): 158. 2005.

Bibliografía: Adler \& Elix (1987).

Amplía su distribución a la provincia de Córdoba. Material estudiado: ARGENTINA. Prov. Córdoba: Dpto. Calamuchita, Camino de los Linderos S 32 04' 23,80"; O 64 51' 58,90", 1620 m, sobre roca, 30-VIII-2013, Rodríguez 2894 (CORD).

Observaciones: especie descubierta en Argentina como Canomaculina tandilensis.

Parmotrema uruguense (Kremp.) Hale, Phytologia 28 (4): 339. 1974.

Bibliografía: Estrabou (1999).

Amplía su distribución as las provincias de Catamarca y Tucumán.

Material estudiado: ARGENTINA. Prov. Catamarca:Dpto. Ambato, Colpes (Río Los Puestos), S $28^{\circ} 02^{\prime} 17,00^{\prime \prime}$; O 65 49 '17,00"', 1037 m, sobre corteza, 12-VII-2011, Rodriguez 2682 (CORD). Prov. Tucumán: Dpto. Chicligasta, RP 365 a $10 \mathrm{~km}$ límite con Catamarca en bosque de

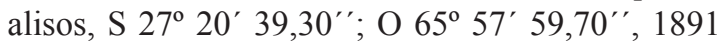
m, sobre Alnus acuminata, 10-VII-2011, Rodríguez 2669 (CORD).

Observaciones: especie citada en Argentina como Rimeliella uruguense.

Parmotrema ventanicum (Adler \& Elix) O. Blanco, A. Crespo, Divakar, Elix \& Lumbsch, Mycologia 97(1): 158. 2005.

Bibliografía: Adler \& Elix (1987).

Amplía su distribución a la provincia de Córdoba. Material estudiado: ARGENTINA. Prov. Córdoba: Dpto. Calamuchita, Camino de los
Linderos,S $32^{\circ} 04^{\prime} 23,80^{\prime \prime}$; O 64 51' 58,90", 1620 m, sobre roca, 30-VIII-2013, Rodríguez 2895 (CORD).

Observaciones: especie descubierta en Argentina como Canomaculina ventanica.

Physcia aipolia (Humb.) Fürnr., Naturhist. Topogr. Regensburg: 249. 1839.

Bibliografía: Moberg (2002).

Amplía su distribución as las provincias de Catamarca y Tucumán.

Material estudiado: ARGENTINA. Prov. Catamarca:Dpto. Ambato-Andalgalá, Las Chacritas (a $10 \mathrm{~km}$ de Singuil), S $27^{\circ} 42^{\prime} 39,40^{\prime \prime}$; O $65^{\circ} 54^{\prime}$ 19,60", 1838 m, sobre Alnus acuminata, 11-VII2011, Rodríguez 2699 (CORD). Prov. Tucumán: Dpto. Yerba Buena, RP 340 Pasando San Javier al lado del camino, S 26 46' 15,60"'; O 65 21' 10,60"', 1180 m, 10-VII-2011, Rodríguez 2547 (CORD).

Physcia krogiae Moberg, Nord. J. Bot. 6(6): 858. 1986.

Bibliografia: Jungbluth (2010).

Amplía su distribución a la provincia de Córdoba. Material estudiado: ARGENTINA. Prov. Córdoba: Dpto. General San Martín, Estancia Yucat, S $32^{\circ} 22^{\prime} 1,68^{\prime \prime}$; O $63^{\circ} 26^{\prime} 13,50^{\prime \prime}$, sobre Prosopis sp., V-2014, Filippini 3099 (CORD).

Physcia tribacia (Ach.) Nyl., Flora: 48. 1874. Bibliografía: Moberg (2002).

Amplía su distribución a la provincia de Catamarca.

Material estudiado: ARGENTINA. Prov. Catamarca: Dpto. Andalgalá, Qda. de Vis-Vis, S $27^{\circ} 23^{\prime} 2,40^{\prime \prime}$; O 66 32' 29,90"', $2017 \mathrm{~m}$, sobre roca, 30-VII-2014, Hernández 07 (CORD).

Placomaronea candelarioides Räsänen, Arch. Soc. Zool. Bot. Fenn. "Vanamo" 20 (3): 29. 1944.

Bibliografía: Westberg et al. (2009).

Amplía su distribución a la provincia de Catamarca.

Material estudiado: ARGENTINA. Prov. Catamarca: Dpto. Andalgalá, Qda. de Vis-Vis, S $27^{\circ} 21^{\prime} 33,30^{\prime \prime}$; O 66 $36^{\circ} 10,80^{\prime \prime}, 2135 \mathrm{~m}$, sobre roca, 30-VII-2014, Hernández 09 (CORD).

Punctelia borrerina (Nyl.) Krog, Nord. J. Bot. 2: 291. 1982. 
Bibliografía: Adler (2013).

Amplía su distribución as las provincias de Catamarca y Tucumán.

Material estudiado: ARGENTINA. Prov. Catamarca: Dpto. Andalgalá, RP 1 al límite con

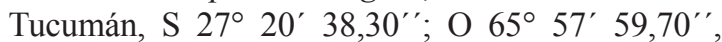
1896 m, sobre Alnus acuminata, 11-VII-2011, Rodríguez 2898 (CORD). Prov. Tucumán: Dpto. Yerba Buena, Camino a San Javier, S $26^{\circ} 48^{\prime}$ 5,10'"; $\mathrm{O} 65^{\circ} 21^{\prime} 2,20^{\prime \prime}, 1007 \mathrm{~m}$, sobre ramas, 09-VII-2011, Rodríguez 2542 (CORD).

Observaciones: especie citada en Argentina como Punctelia microsticta.

Punctelia constantimontium Sérus. Nord. J. Bot. 3 (4): 517. 1983.

Bibliografía: Spielmann \& Marcelli (2008).

Amplía su distribución a la provincia de Tucumán.

Material estudiado: ARGENTINA. Prov.

Tucumán: Dpto. Tafi, RP 307 pasando Reserva Los

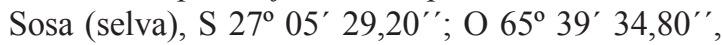
723 m, sobre rama, 10-VII-2011, Rodríguez 2661 (CORD).

Punctelia punctilla (Hale) Krog, Nord. J. Bot. 2 (3): 291. 1982.

Bibliografía: Adler \& Calvelo (2010).

Amplía su distribución a la provincia de Catamarca.

Material estudiado: ARGENTINA. Prov. Catamarca: Dpto. Ambato. Sierra de Ambato, S $28^{\circ}$ $00^{\prime} 41,20^{\prime \prime}$; O $65^{\circ} 56^{\prime} 33,10^{\prime \prime}, 1684 \mathrm{~m}$, sobre roca, 15-X-2007, Rodríguez 2691 (CORD).

Punctelia semansiana (W.L. Culb. \& C.F.Culb.)

Krog, Nord. J. Bot. 2 (3): 291. 1982.

Bibliografía: Estrabou (1999).

Amplía su distribución a la provincia de Catamarca.

Material estudiado: ARGENTINA. Prov. Catamarca: Dpto. Andalgalá, Qda. de Vis-Vis, S $27^{\circ}$ $27^{\prime} 22,47^{\prime \prime}$; O 66 31' 18,90'", 1700 m, sobre Larrea divaricata, 13-VIII-2015, Rodríguez \& Hernández 3131 (CORD).

Punctelia stictica (Delise ex Duby) Krog Nord. J. Bot. 2: 291. 1982.

Bibliografía: Adler \& Calvelo (2007).

Amplía su distribución a la provincia de Catamarca.
Material estudiado: ARGENTINA. Prov. Catamarca: Dpto. Ambato. Sierra de Ambato, S $28^{\circ} 08^{\prime} 20,40^{\prime \prime}$; O $65^{\circ} 53^{\prime} 31,00^{\prime \prime}, 1617 \mathrm{~m}$, sobre Acacia caven, 15-X-2007, Rodríguez 2030 (CORD).

Pyxine berteriana (Fée) Imshaug, Trans. Amer. Microscop. Soc. 76 (3): 254. 1957.

Bibliografía: Aptroot et al. (2014).

Amplía su distribución a la provincia de Tucumán. Material estudiado: ARGENTINA. Prov. Tucumán: Dpto. Yerba Buena, RP 340 Pasando San Javier al lado del camino, S $26^{\circ} 46^{\prime} 15,60^{\prime \prime}$; O $65^{\circ} 21^{\prime} 10,60^{\prime \prime}, 1180 \mathrm{~m}$, sobre rama, 10-VII-2011, Rodríguez 2552 (CORD).

Pyxine subcinerea Stirt., Trans. \& Proc. New Zealand Inst. 30: 397. 1898.

Bibliografía: Aptroot et al. (2014).

Amplía su distribución a la provincia de Catamarca.

Material estudiado: ARGENTINA. Prov. Catamarca: Dpto. Ambato, Colpes (Río Los

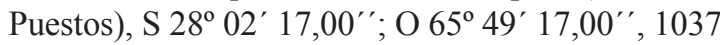
$\mathrm{m}$, sobre corteza, 10-VII-2011, Rodríguez 2562 (CORD).

Ramalina celastri (Spreng.) Krog \& Swinscow, Norweg. J. Bot. 23 (3): 159. 1976.

Bibliografía: Kashiwadani \& Kalb (1993).

Amplía su distribución a la provincia de Catamarca.

Material estudiado: ARGENTINA. Prov. Catamarca: Dpto. Ambato, La Calera, S 28 22' 12,90"'; O 65 52' 10,40"', $1154 \mathrm{~m}$, sobre corteza, 10-VII-2011, Rodríguez 2679 (CORD).

Ramalina peruviana Ach. Lichenogr. Universalis: 599. 1810.

Bibliografía: Kashiwadani \& Kalb (1993).

Amplía su distribución a la provincia de Catamarca.

Material estudiado: ARGENTINA. Prov. Catamarca: Dpto. Santa Rosa, a $10 \mathrm{~km}$ de Bañado de Ovanta (dirección este), S $28^{\circ} 07^{\prime} 46,30^{\prime \prime}$; O $65^{\circ} 15^{\prime} 47,10^{\prime \prime}, 523 \mathrm{~m}$, sobre rama, 12-VII-2011, Rodríguez 2614 (CORD).

Sticta fuliginosa (Dicks.) Ach., Methodus (Acharius): 280. 1803. 
Bibliografía: Galloway (1994).

Amplía su distribución a la provincia de Catamarca.

Material estudiado: ARGENTINA, Prov. Catamarca: Dpto. Andalgalá, cuenca del río Potrero, S $27^{\circ} 25 \square 47,90 \square \square$; O 66 $6^{\circ} 17 \square 39,50 \square \square$, $2178 \mathrm{~m}$, sobre roca, 28-VII-2014, Hernández 26 (CORD).

Sticta weigelii (Ach.) Vain., Lichenogr. Universalis: 446. 1810.

Bibliografía: Galloway (1994).

Amplía su distribución a la provincia de Catamarca.

Material estudiado: ARGENTINA. Prov. Catamarca: Dpto. Paclin, a $10 \mathrm{~km}$ de La Merced, $\mathrm{S} 28^{\circ} 06^{\prime} 1,60^{\prime \prime}$; O $65^{\circ} 36^{\prime} 45,90^{\prime \prime}, 983 \mathrm{~m}, 12-\mathrm{VII}-$ 2011, Rodríguez 2588 (CORD).

Teloschistes chrysophthalmus (L.) Beltr., Lich. Bassan.: 109. 1858.

Bibliografía: Almborn (1992).

Amplía su distribución a la provincia de Catamarca.

Material estudiado: ARGENTINA. Prov. Catamarca: Dpto. Andalgalá, Narvaez S $27^{\circ} 39^{\prime}$ 02,4"', O 65 56' 47,4", 1948 m, 11-VII-2011, Rodríguez 2690 (CORD).

Teloschistes cymbalifer (G. Mey.) Müll. Arg., Rev. Mycol. (Toulouse) 10: 2. 1888.

Bibliografía: Almborn (1992).

Amplía su distribución a las provincias de Catamarca y Tucumán.

Material estudiado: ARGENTINA. Prov. Catamarca: Dpto. Valle Viejo, Portezuelo, S $28^{\circ} 27^{\prime}$ 28,20"'; O 65 37' 37,20"', 647 m, 12-VII-2011, Rodríguez 2576 (CORD). Prov. Tucumán: Dpto. Yerba Buena, Reserva San Javier, pasando San Javier en bosque de Eucaliptus, S 26 46' 15,62'"; O $65^{\circ} 21^{\prime} 10,60^{\prime \prime}$, sobre rama, 09-VII-2011, Rodríguez 2677 (CORD).

Teloschistes flavicans (Sw.) Norman, Nytt Mag. Naturvidensk. 7: 229. 1853.

Bibliografía: Almborn (1992).

Amplía su distribución a la provincia de Tucumán.

Material estudiado: ARGENTINA. Prov. Tucumán: Dpto. Chicligasta, límite Tucumán-
Catamarca (cuesta del Clavillo), S $27^{\circ} 20^{\prime} 2,00^{\prime \prime}$; O $65^{\circ} 56^{\prime} 52,80^{\prime \prime}, 1790 \mathrm{~m}$, sobre aliso, 11-VII-2011, Rodríguez 2677 (CORD).

Teloschistes hosseusianus Gyeln., Borbásia nova: 1.1942.

Bibliografía: Almborn (1992).

Amplía su distribución a la provincia de Catamarca.

Material estudiado: ARGENTINA. Prov. Catamarca: Dpto. Valle Viejo, Portezuelo, S $28^{\circ} 27^{\prime}$ 28,20"'; O $65^{\circ} 37^{\prime} 37,20^{\prime \prime}, 674$ m, 12-VII-2011, Rodríguez 2596 (CORD).

Teloschistes nodulifer (Nyl.) Hillmann, Hedwigia 69: 326. 1930.

Bibliografía: Almborn (1992).

Amplía su distribución a la provincia de San Luis.

Material estudiado: ARGENTINA. Prov. San Luis:Dpto. Junín, Final RP 5, sobre musgo y roca, Rodriguez 2717 (CORD).

Usnea alata Motyka, Lich. Gen. Usnea Stud. Monogr. Pars Syst. 2: 395. 1938.

Bibliografía: Truong et al. (2013).

Amplía su distribución a la provincia de Santiago del Estero.

Material estudiado: ARGENTINA. Prov. Santiago del Estero: Dpto. Copo, Parque Nacional

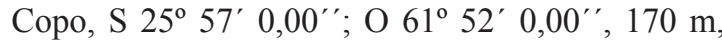
sobre ramas secas, 10-VII-2009, Dominguez 1205 (CORD).

Usnea amblyoclada (Müll. Arg.) Zahlbr., Cat. Lich. Univ. 6: 534. 1930

Bibliografía: Rodríguez \& Estrabou (2008).

Amplía su distribución a la provincia de Tucumán.

Material estudiado: ARGENTINA. Prov. Tucumán: Dpto. Tafí, El Infiernillo, Faltan coordenadas, $1900 \mathrm{~m}$, Grundlehner 51.28.3 (CORD).

Usnea angulata Ach. Synops. Lich. 307. 1814.

Bibliografía: Truong et al. (2013).

Amplía su distribución a la provincia de Santiago del Estero.

Material estudiado: ARGENTINA. Prov. Santiago del Estero: Dpto. Guasayán, Sierras de 


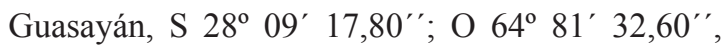
491 m, 19-III-2008, Estrabou \& Rodríguez 0434 (CORD).

Usnea cristatula Motyka, Lich. Gen. Usnea Stud. Monogr., Pars Syst 2: 641. 1936-1938.

Bibliografía: Truong et al. (2013).

Amplía su distribución a la provincia de Córdoba.

Material estudiado: ARGENTINA. Prov. Córdoba: Dpto. Tulumba, Mar Chiquita, Miramar, sobre Schinus, 13-XII-2004, Rodríguez 839 (CORD).

\section{Usnea dasaea Stirt., Scott. Naturalist (Perth) 6:} 104. 1881.

Bibliografía: Clerc \& Herrera-Campos (1997).

Amplía su distribución as las provincias de Catamarca, Santiago del Estero y Tucumán.

Material estudiado: ARGENTINA. Prov. Catamarca: Dpto. Ambato, Vuelta de Ambato, S $28^{\circ}$ 03' 59,60"; O 65 54' 22;00"', 1646 m, Rodríguez 0223 (CORD). Prov. Santiago del Estero: Dpto. Guasayán, Sierras de Guasayán, S $28^{\circ} 09^{\prime} 17 ; 80^{\prime \prime}$; O 64 81' 32,60", 491 m, Estrabou \& Rodríguez 0435 (CORD. Prov. Tucumán: Dpto. Yerba Buena, Reserva San Javier, S $26^{\circ} 46^{\prime} 28,00^{\prime \prime}$; O 65 20' 0,20", 773 m, Rodríguez 0145 (CORD).

Usnea durietzii Motyka, Lich. Gen. Usnea Stud. Monogr., Pars Syst. 2: 503. 1936-1938.

Bibliografía: Rodríguez et al. (2011).

Amplía su distribución a la provincia de Catamarca.

Material estudiado: ARGENTINA. Prov. Catamarca: Dpto. Andalgalá, cuenca del río Potrero, S $27^{\circ} 25^{\prime}$ 47,90"'; O 66 17' 39,50'", 2178 $\mathrm{m}$, sobre roca, Hernández 17 (CORD).

Observaciones: incluye a su sinónimo Usnea caespitia.

Usnea parvula Motyka, Lich. Gen. Usnea Stud. Monogr. Pars Syst. 2: 599 (1936-1938).

Bibliografía: Rodríguez (2011).

Amplía su distribución a las provincias de Catamarca, La Rioja, Santiago del Estero y Tucumán.

Material estudiado: ARGENTINA. Prov. Catamarca: Dpto. Ambato, Sierra de Ambato, antes de Piedras Blancas, 3500-4000 m, sobre poste, Pignata 600 (CORD). Prov. La Rioja: Dpto.
Famatina, Cuesta La Agüadita, $20 \mathrm{~km}$ de Famatina, S $28^{\circ} 44^{\prime} 30,10^{\prime \prime}$; O 67 35' 40,60", $2059 \mathrm{~m}$, sobre Condalia microphylla, 14-X-2007, Rodríguez 222 (CORD). Prov. Santiago del Estero: Dpto. Guasayán, Sierras de Guasayán, S 28 09' 15,00"'; O $64^{\circ} 80^{\prime} 29,60^{\prime \prime}, 463$ m, sobre Schinopsis lorentzii, 19-III-2008, Estrabou 447 (LIL). Prov. Tucumán: Dpto. Tafí, El Infiernillo, km 487, Grundlehner 19283 (CORD).

Usnea perplectata Motyka, Lich. Gen. Usnea Stud. Monogr., Pars Syst. 2: 55. 1936-1938.

Bibliografía: Truong \& Clerc (2012).

Amplía su distribución a la provincia de Catamarca.

Material estudiado: ARGENTINA. Prov. Catamarca: Dpto. Ambato, Vuelta de Ambato, S $28^{\circ}$ 03' 59,60"; O 65 54' 22,0"', 1646 m, 14-X-2007, Rodríguez 231 (CORD).

Usnea rubicunda Stirt., Scott. Naturalist 6: 102. 1881.

Bibliografía: Truong \& Clerc (2012).

Amplía su distribución a las provincias de Córdoba y Tucumán.

Material estudiado: ARGENTINA. Prov. Córdoba: Dpto. Colón, La Quebrada, camino a cascada de los Hornillos, sobre Condalia montana, 29-V-2004, Rodríguez 922 (CORD). Prov. Tucumán: Dpto. Tafí, Tafí del Valle, 2000 m, 12-XI-1946, O'Donell 7284 (CORD).

Usnea steineri Zahlbr. Denkschr. Kaiserl. Akad. Wiss., Wien. Math.-Naturwiss. Kl., 83: 183-186. 1909.

Amplía su distribución a las provincias de Catamarca, Santiago del Estero y Tucumán.

Bibliografía: Truong et al. (2011).

Material estudiado: ARGENTINA. Prov. Catamarca: Dpto. Ambato, Vuelta de Ambato, S $28^{\circ}$ 03' 59,60"' O 65 54' 22,00"', 1646 m, sobre Acacia caven, 14-X-2007, Rodríguez 1126 (CORD). Prov. Santiago del Estero: Dpto. Guasayán, Sierras de Guasayán, Maquijata, S $27^{\circ} 14^{\prime} 49,30^{\prime \prime}$; O 64 $25^{\circ}$ 47,90", 463 m, sobre Prosopis nigra, Rodríguez \& Estrabou 0444 (CORD). Prov. Tucumán: Dpto. Yerba Buena, Reserva San Javier, S $26^{\circ} 46^{\prime} 0,50^{\prime \prime}$; O $65^{\circ}$ 19' 71,00", 723 m, sobre Cederella sp., 05-VI-2006, Rodríguez 158 (CORD).

Observaciones: esta especie incluye a su sinónimo Usnea krempelhuberi. 
Usnea strigosa (Ach.) Eaton, Bot. North. Middle States: 431.1829.

Bibliografía: Rodríguez (2011).

Amplía su distribución a las provincias de Catamarca, Santiago del Estero y Tucumán.

Material estudiado: ARGENTINA. Prov. Catamarca: Dpto. Ambato, Vuelta de Ambato, S $27^{\circ} 51^{\prime}$ 51,30'"; O 65 55' 3,90'", 1660 msnm, sobre Acacia caven, 14-X-2007, Rodríguez 0266 (CORD). Prov. Santiago del Estero: Dpto. Capital, Pampa, Muyaj, S $27^{\circ}$ 53' 46,60"'; O 64 25' 47,90", $269 \mathrm{msnm}$, sobre Larrea divaricata, 19-III-2008, Rodríguez 0446. Prov. Tucumán:Dpto. Tafi, camino a Ampimpa, S 26 42' 22,90'"; O 65 47' 43;90", 299 m, Hladki 4013 (CORD).

Usnea subelegans (Vain.) de Lesd., Ann. Cryptog. Exot. 6: 112.1933.

Bibliografía: Rodríguez (2011).

Amplía su distribución a las provincias de Córdoba y Tucumán.

Material estudiado: ARGENTINA. Prov. Córdoba: Dpto. Punilla, Los Gigantes, S $31^{\circ} 25^{\prime}$ 39,50"'; O 64 45' 48,60" $1682 \mathrm{msnm}$, sobre poste, III-2004, Rodríguez 0513 (CORD). Prov. Tucumán: Dpto. Tafi, camino a Ampimpa, S $26^{\circ} 42^{\prime} 22,90^{\prime \prime}$; O 65 47' 43,90'", 299 m, Hladki 4013 (CORD).

Xanthoparmelia cotopaxiensis T.H. Nash III, Elix \& J. Johnst., Mycotaxon 28 (2): 288. 1987.

Bibliografía: Nash et al. (1995).

Amplía su distribución a la provincia de Catamarca.

Material estudiado: ARGENTINA. Prov. Catamarca: Dpto. Andalgalá, Narvaez, S $27^{\circ} 39^{\prime}$ 2,40"'; O 65 56' 47,40"', $1948 \mathrm{~m}$, sobre roca, 11VII-2011, Rodríguez 2594 (CORD).

Xanthoparmelia ferraroiana T.H. Nash III, Elix \& J. Johnst., Mycotaxon 28 (2): 289. 1987.

Bibliografía: Nash et al. (1995).

Amplía su distribución a la provincia de Catamarca.

Material estudiado: ARGENTINA. Prov. Catamarca: Dpto. Andalgalá. Qda. de Vis-Vis, S $27^{\circ} 21^{\prime} 33,30^{\prime \prime}$; O 66 $33^{\circ} 10,80^{\prime \prime}, 2135 \mathrm{~m}$, sobre roca, 30-VII-2014, Hernández 18 (CORD).

Xanthoparmelia hypopsila (Müll. Arg.) Hale, Phytologia 28 (5): 488. 1974.
Bibliografía: Nash et al. (1995).

Amplía su distribución a la provincia de Catamarca.

Material estudiado: ARGENTINA. Prov. Catamarca: Dpto. Andalgalá, cuenca del río

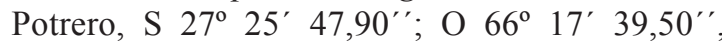
$2178 \mathrm{~m}$, sobre roca, 30-VII-2014, Hernández 19 (CORD).

Xanthoparmelia imitatrix (Taylor) O. Blanco, A. Crespo, Elix, D. Hawksw. \& Lumbsch, Taxon 53 (4): 968. 2004.

Bibliografía: Calvelo (1994).

Amplía su distribución a la provincia de Córdoba. Material estudiado: ARGENTINA. Prov. Córdoba: Dpto. Calamuchita, Camino de los Linderos,S $32^{\circ} 04^{\prime}$ 23,80"'; O 64 51' 58,90", $1620 \mathrm{~m}$, sobre roca, 30-VIII-2013, Rodríguez 2884 (CORD).

Observaciones: especie citada en Argentina como Neofuscelia imitatrix.

Xanthoparmelia scabrosa (Taylor) Hale, Phytologia 28 (5): 488. 1974.

Bibliografía: Nash et al. (1995).

Amplía su distribución a la provincia de Catamarca.

Material estudiado: ARGENTINA. Prov. Catamarca: Dpto. Andalgalá, Narvaez, S 27 39' 2,40"'; O 650 56' 47,40"', $1948 \mathrm{~m}$, sobre roca, 11 VII-2011, Rodríguez 2680 (CORD).

Xanthoparmelia wrightiana T.H. Nash III, Elix \& J. Johnst., Mycotaxon 28 (2): 290. 1987.

Bibliografía: Nash et al. (1995).

Amplía su distribución a la provincia de Catamarca.

Material estudiado: ARGENTINA. Prov. Catamarca: Dpto. Andalgalá. Qda. de Vis-Vis, DS6, S $27^{\circ} 27^{\prime} 22,47^{\prime \prime}$; O $66^{\circ} 31^{\prime} 18,90^{\prime \prime}, 1700$ $\mathrm{m}$, sobre suelo, 10-VIII-2015, Rodríguez 3122 (CORD).

\section{Agradecimientos}

El primer autor agradece a la Myndel Botanica Foundation por los fondos recibidos para financiar viajes de colección a las provincias del centro de Argentina. A los revisores anónimos por las 
sugerencias. Los autores JMH y EF son becarios de CONICET y JMR becario de SECyT - UNC.

\section{Biblografía}

ADLER, M. T. 2013. Líquenes Parmelioides (Parmeliaceae, Ascomycota) del Parque Nacional Copo (Provincia de Santiago del Estero, Argentina). Bol. Soc. Argent. Bot. 48: 387-406.

ADLER, M. T. \& J. A. ELIX. 1987. Three new saxicolous species in Parmeliaceae (lichenized Ascomycotina) from Argentina. Mycotaxon 30: 339-344.

ADLER, M. T. \& J. A. ELIX. 1992. New records of Hypotrachyna and Parmelinopsis lichens (Ascomycotina, Parmeliaceae) from north-west and central Argentina. Mycotaxon 43: 283-288.

ADLER, M. T. \& S. CALVELO. 2007. Ampliación de las distribuciones de especies de Parmeliaceae (Ascomycota liquenizados) en la República Argentina. Bol. Soc. Argent. Bot. 42: 1-11.

ADLER, M. T. \& S. CALVELO. 2010. Flavoparmelia baltimorensis, Parmotrema yodae y Xanthoparmelia braziliensis, primeras citas para Argentina y ampliación de distribuciones para otras Parmeliaceae (Ascomycota liquenizados). Bol. Soc. Argent. Bot. 45: 5-16.

ALMBORN, O. 1992. Some overlooked or misidentified species of Teloschistes from South America and a key to the South-American species. Nord. J. Bot. 12: 361-364.

APTROOT, A., JUNGBLUTH, P. \& M. E. S. CÁCERES. 2014. A world key to the species of Pyxine with lichexanthone, with a new species from Brazil. Lichenologist 46: 669-672.

CABRERA, A. L. 1971. Fitogeografía de la República Argentina. Bol. Soc. Argent. Bot. 14: 1-42.

CALVELO, S. 1994. Parmeliaceae s. lat. (Ascomycetes liquenizados) foliosos de los bosques andinopatagónicos y de Tierra del Fuego: estudios taxonómico-florísticos. Tesis de Doctorado. Universidad de Buenos Aires. Argentina. Inédita.

CALVELO, S. \& C. ESTRABOU. 1997. The genus Cetrariastrum in southern South America and $C$. billingsii as a taxonomic synonym of $C$. americanum. Lichens 1: 11-17.

CALVELO, S. \& S. LiBERATORE. 2002. Catálogo de los líquenes de la Argentina. Kurtziana 29 (2): 7-170.

CLERC, P. \& M. A. HERRERA-CAMPOS. 1997. Saxicolous species of Usnea subgenus Usnea (lichenized Ascomycetes) in North America. Bryologist 100 : 281-301.

ESTRABOU, C. 1999. La familia Parmeliaceae (s. str.) en la provincia de Córdoba. Análisis sistemático y biogeográfico. Tesis Doctorado en Ciencias Biológicas, Universidad Nacional de Córdoba, inédita.

ESTRABOU, C., RODRÍGUEZ, J. M., PRIERI, B. \& R. LIJTEROFF. 2006. Contribución al conocimiento de los macrolíquenes del extremo Sur del Gran Chaco (Argentina). Kurtziana 32(1-2): 25-43.

FERRARO, L. I. \& A. MICHLIG. 2013. New species and additional records of Coenogonium (Ostropales: Coenogoniaceae) from southern South America. Lichenologist 45: 497-504.

FILIPPINI, E., QUIROGA, G., RODRÍGUEZ, J. M., \& C. ESTRABOU. 2015. The genus Hyperphyscia Müll. Arg. (Physciaceae, Ascomycota) in Argentina. Sydowia 67: 22- 32.

GALLOWAY, D. J. 1994. Studies on the lichen genus Sticta (Schreber) Ach.: I. Southern South American species. Lichenologist 26: 223-282.

GBIF. 2015. Global Biodiversity Information Facility [online]. Disponible en: http://www.gbif.org/ [Acceso: Octubre 2015].

JUNGBLUTH, P. 2010. Estudos taxonômicos em Physcia (Schreb.) Michx. e Pyxine Fr. (Physciaceae, Ascomycota). Tesis doctoral. Doctorado en Biodiversidad Vegetal y Medio Ambiente. Instituto de Botânica da Secretaria de Estado do Meio Ambiente.

KASHIWADANI, H. \& K. KALB. 1993. The genus Ramalina in Brazil. Lichenologist 25: 1-31.

LENDEMER, J. C. \& R. YAHR, R. 2004. Changes and additions to the checklist of North American lichens II. Mycotaxon 90: 319-322.

MICHLIG, A. 2013. Estudios taxonómicos de la familia Parmeliaceae sensu stricto (Ascomycota liquenizados) del Nordeste de Argentina. Tesis doctoral. Doctorado en Ciencias Biológicas. Universidad Nacional de Córdoba. Inédita.

MICHLIG, A. 2014. Canoparmelia y Crespoa (Parmeliaceae, Ascomycota) en el nordeste de Argentina; Canoparmelia caroliniana y $C$. cryptochlorophaea nuevas citas para Argentina. Bol. Soc. Argent. Bot. 49: 161-172.

MICHLIG, A. \& L. I. FERRARO. 2012. Nuevos registros de especies sorediadas de Parmotrema con ácido protocetrárico (Ascomycota, Parmeliaceae) en el sur de Sudamérica. Darwiniana 50: 323-331.

MOBERG, R. 2002. Physcia. In: NASH III, T.H., RYAN, B. D., GRIES, C. \& F. BUNGARTZ (eds.), Lichen Flora of the Greater Sonoran Desert Region. I. Lichens Unlimited, pp. 358-373. Arizona State University, Tempe.

NASH III, T. H., C. GRIES, \& J. A. ELIX. 1995. A revision of the lichen genus Xanthoparmelia in South America. Bibl. Lichenol. 56: 1-157.

OBERMAYER, W., KALB, K., SIPMAN H. J. M. \& T. H. NASH III. 2009. New reports of Culbersonia 


\section{J. M. Rodriguez et al. - Nuevas citas de macrolíquenes para Argentina}

nubila (Moberg) Essl. from the Tibetan Region, Bolivia, Argentina, Lesotho and South Africa. Lichenologist 41: 683-687.

ORANGE, A., P. W. JAMES \& F. J. WHITE. 2010. Microchemical methods for the identification of lichens. 2 nd ed. British Lichen Society, London.

PASSO, A. \& S. CALVELO. 2006. New reports and combinations in the family Pannariaceae (Lecanorales, lichenized Ascomycota). Lichenologist 38: 549-555.

PASSO, A., S. STENROOS \& S. CALVELO. 2008. Joergensenia, a new genus to accommodate Psoroma cephalodinum (lichenized Ascomycota). Mycol. Res. 112: 1465-1474.

RODRÍGUEZ, J. M. \& C. ESTRABOU. 2008. Usnea ambyloclada "barba de piedra" (Ascomycetes liquenizados) en Argentina. Bol. Soc. Argent. Bot. 43: 221-225.

RODRÍGUEZ, J. M., ESTRABOU, C., TRUONG, C. \& P. CLERC. 2011. The saxicolous species of the genus Usnea subgenus Usnea (Parmeliaceae) in Argentina and Uruguay. Bryologist 114: 504-525.

RODRÍGUEZ, J. M., ESTRABOU, C., \& G. QUIROGA. 2012. The genus Heterodermia (Lecanorales). Acta Bot. Bras. 26: 1000-1005.

SPIELMANN, A. A. \& M. P. MARCELLI. 2008. Punctelia (Parmeliaceae, lichenized Ascomycota) from roadsides and slopes in the Serra Geral of Rio Grande do Sul, Brazil Biociências 16: 79-91.
TAVARES, I. I. 1987. The taxa of Usnea (Lichenes) described by Michaux from eastern North America. Mycotaxon 30: 39-68.

TRUONG, C., BUNGARTZ, B. \& P. CLERC. 2011. The lichen genus Usnea (Parmeliaceae) in the tropical Andes and the Galapagos: species with a red-orange cortical or subcortical pigmentation. Bryologist 114: 477-503.

TRUONG, C. \& P. CLERC. 2012: The lichen genus Usnea (Parmeliaceae) in tropical South America: species with a pigmented medulla, reacting $\mathrm{C}+$ yellow. Lichenologist 44: 625-637.

TRUONG, C., RODRIGUEZ, J. M. \& P. CLERC. 2013. Pendulous Usnea species (Parmeliaceae, lichenized Ascomycota) in tropical South America and the Galapagos. Lichenologist 45: 505-542.

WESTBERG, M. \& T. H. NASH III. 2002. Candelaria. In: NASH III, T. H., B. D. RYAN, GRIES, C. \& F. BUNGARTZ (eds.), Lichen Flora of the Greater Sonoran Desert Region. Vol I. Lichens Unlimited, Arizona State University, pp. 116-118. Tempe.

WESTBERG, M., P. FRÖDÉN, \& M. WEDIN. 2009. A monograph of the genus Placomaronea (Ascomycota, Candelariales). Lichenologist 41: 513-527.

Recibido el 31 de diciembre de 2015, aceptado el 6 de mayo de 2016. 
